\title{
Blood lipids and the treatment of essential hypertension with methyldopa and bendrofluazide
}

\author{
L. J. LIBMAN* \\ B.SC., M.R.C.P. \\ T. L. DORMANDY \\ PH.D., F.R.C.S. (Ed.), M.C. Path.
}

\author{
D. E. Arrowsmith \\ F.I.M.L.T. \\ JOANNA HEAL $\dagger$ \\ M.R.C.P.
}

\author{
B. I. HOFFBRAND \\ D.M., M.R.C.P.
}

Departments of Medicine and Chemical Pathology, Whittington Hospital, London N.19

\begin{abstract}
Summary
The blood lipids of eight patients with previously untreated essential hypertension have been studied before and after treatment with methyldopa and bendrofluazide. No significant change was found in any of the measurements over a mean treatment period of 5.25 months. These results are believed to provide evidence that hypotensive therapy does not predispose to the development of ischaemic heart disease.
\end{abstract}

\section{Introduction}

The treatment of hypertension has resulted in a change in the incidence of the major causes of death attributable to this condition. There has been a fall in the percentage of patients dying of cerebrovascular disease, renal failure and heart failure but an increase in the numbers dying from coronary artery disease (Breckenridge, Dollery and Parry, 1970). It is not clear from the available data whether or not the increase in the incidence of deaths from coronary artery disease is due to the reduction in deaths from the other causes or whether hypotensive therapy itself may be more directly responsible.

Numerous epidemiological studies have shown that blood lipid levels correlate strongly with the development of clinical ischaemic heart disease (IHD). The relationship between IHD and serum cholesterol extends throughout the entire spectrum of serum cholesterol values. Lipid metabolism and blood lipid levels are influenced to a significant extent by the sympathetic nervous system and circulating catecholamines (Havel, 1968). It is

\footnotetext{
* Present address: Senior Medical Registrar, University College Hospital, London W.C.1.

$\dagger$ Present address: Research Assistant, Georgetown University, Washington D.C., U.S.A.

Correspondence: Dr B. I. Hoffbrand, Archway Wing, Whittington Hospital, Archway Road, London N.19.
}

apparent that drugs which interfere with the sympathetic nervous system could adversely affect blood lipid levels and hence predispose to clinical IHD. There have been few detailed studies of blood lipid levels during treatment of hypertension. We have studied the lipid profiles of a group of hypertensive patients before and during methyldopa and bendrofluazide therapy using some more recently introduced methods of lipoprotein analysis.

\section{Patients and methods}

Eight patients were investigated There were six males and two females with an age range of 31-68 years. All the subjects had significant hypertension with an erect diastolic blood pressure of $110 \mathrm{mmHg}$ or more on three separate occasions. No patient had previously received any therapy for hypertension and none was obese or had clinical evidence of atherosclerosis. Investigation revealed that the patients had essential hypertension with normal renal function. There was no clinical or biochemical evidence of diabetes mellitus, myxoedema, nephrotic syndrome or gout.

The following serum lipids were analysed. Cholesterol was measured on the autoanalyser using 'Technicon' Method N 24a. Triglycerides were measured on an autoanalyser using the method of Noble and Campbell (1970) as modified by McLellan (1971). Total lipids were measured by the method of Woodman and Price (1972). Lipoprotein electrophoresis was performed on cellulose acetate which was stained with 'Oil red O' (Dangerfield and Tarlow, 1971). The fractions were quantitated visually. The concentration of $\mathbf{S}, \mathbf{M}$ and $\mathbf{L}$ particles was estimated nephelometrically as described by Stone and Thorpe (1966). 'Lipoprotein profiles' were based on the classification of Fredrickson but taking the S-M-L scores into account (Fredrickson, Levy and Lees, 
1967; Bulletin of the World Health Organization, 1970). Free fatty acids were measured by the method of Dole (1956) as modified by Trout, Estes and Friedberg (1960). Glucose was measured by a glucose oxidase autoanalyser method, and when an intravenous glucose tolerance test was performed the results were expressed as the $\mathrm{K}$-value-the slope of the blood-sugar curve in a semi-logarithmic system (Lundbaek, 1962).

Two control blood samples were taken on separate days with the patients either in hospital or outpatients, no dietary advice being given at any stage. They fasted overnight and rested supine for $30 \mathrm{~min}$ before the samples were taken between 8.30 a.m. and $9.30 \mathrm{a} . \mathrm{m}$. The specimens were analysed for the various lipids mentioned above, and on one of the two occasions an intravenous glucose tolerance test was performed, blood being taken at the same time for free fatty acid measurements, the sera being frozen until analysis. Treatment was then started with methyldopa and the dose changed to obtain blood pressure control (mean dose $2.2 \mathrm{~g}$; range 1.25 $2.5 \mathrm{~g}$ ) At this time (mean time after starting drug, 12 weeks; range 6-22 weeks), the lipid studies were again performed under the same conditions as previously described. Following this a diuretic (bendrofluazide $5 \mathrm{mg}$ or $10 \mathrm{mg}$ daily) was added. Four to 16 weeks after this (mean 9 weeks) the lipid measurements were made for a final time with a second intravenous glucose tolerance test. Following these measurements, the serum cholesterol alone was estimated 24-40 weeks (mean 32 weeks) later.

\section{Results}

The results are shown in Table 1. As will be seen, the blood pressure control was good when the patients were on methyldopa alone or in combination with bendrofluazide. The values of the various blood measurements before and during treatment were compared using a paired ' $t$ ' test. No statistical difference was found between the control lipid measurements nor between the mean control values and those on therapy. The lipoprotein electrophoresis which was quantitated visually also showed no significant changes before or during therapy. The $\mathrm{K}$-value derived from the intravenous glucose tolerance test fell when the patients were taking methylldopa and bendrofluazide. This may represent the diabetogenic effect of thiazide diuretics; Lundbaek (1962) found the K-value to be between 0.20 and 1.02 in a group of diabetics and 0.96 to 3.45 in normals.

The free fatty acids fall during a glucose tolerance test (Hales and Randle, 1963) and this fall did not show any significant change after therapy with methyldopa.
The plasma cholesterol alone was estimated at a mean time of 32 weeks after the final lipid measurements were made, giving a mean value of $212 \mathrm{mg}$ / $100 \mathrm{ml}$ (s.e.m. $15 \mathrm{mg} / 100 \mathrm{ml}$ ), which did not show a significant change.

\section{Discussion}

Hutchinson (1966) found no change in blood lipids in patients treated with methyldopa and a diuretic, but he did not study lipoprotein electrophoretic patterns or use nephelometry. Gross (1965) found a significant fall in fatty acid esters on treatment with this combination of drugs. Our results are in agreement with those of Hutchinson although, in addition, we have found no evidence of any change in the lipoprotein pattern or the response of the free fatty acid levels to an intravenous glucose tolerance test.

The effects of catecholamines and the sympathetic nervous system on carbohydrate and fat metabolism have proved difficult to categorize conclusively in terms of $\alpha$ and $\beta$ adrenergic receptor activation. Lipolysis in adipose tissue is, however, clearly mediated by $\beta$ receptor stimulation (Jenkinson, 1973). We studied three further patients with essential hypertension treated with propranolol and bendrofluazide with an identical experimental protocol. There was no suggestion in any case of a deterioration in blood lipid levels or lipoprotein pattern during effective therapy for several months.

The evidence available suggests that the treatment of hypertension is not associated with significant changes in fasting blood lipids. However, the studies to date have been few, mainly involving methyldopa and diuretics, and over relatively short periods of time. The possibility remains, moreover, that hypotensive therapy predisposes to IHD by some mechanism other than the production of blood lipid abnormalities. It seems likely, however, that increased coronary deaths in treated hypertension merely reflect the reduced incidence of other causes of death in a population with established IHD.

\section{Acknowledgment}

Our thanks are due to $\mathrm{Mr}$ L. Freedman for statistical advice.

\section{References}

Breckenridge, A., Dollery, C.T. \& Parry, E.H.O. (1970) Prognosis of treated hypertension. Quarterly Journal of Medicine, New Series, xxxix, 155, 411.

Bulletin of the World Health Organization (1970) Classification of hyperlipidaemias and hyperlipoproteinaemias. 43, 891.

Dangerfield, W.G. \& Tarlow, D. (1971) Electrophoresis of plasma lipoproteins. Proceedings of the Royal Society of Medicine, 64, 896.

Dole, V.P. (1956) A relation between non-esterified fatty acids in plasma and the metabolism of glucose. Journal of Clinical Investigation, 35, 150. 
Fredrickson, D.S., Levy, R.I. \& LeES, R.S. (1967) Fat transport in lipoproteins-an integrated approach to mechanisms and disorders. New England Journal of Medicine, 276, 34, 94, 148.

Gross, W. (1965) The behaviour of certain serum lipids during the treatment of hypertension. Verhandlungen der Deutschen Gesellschaft für innere Medizin, 71, 441.

Hales, C.N. \& RANDLE, R.J. (1963) Effects of low-carbohydrate diet and diabetes mellitus on plasma concentrations of glucose, non-esterified fatty acid and insulin during oral glucose tolerance tests. Lancet, i, 790.

HAVEL, R.J. (1968) The autonomic nervous system and intermediary carbohydrate and fat metabolism. Anesthesio$\log y, 29,702$.

Hutchinson, J.C., VANDerbeck, R.R. \& Roediger, P.M. (1966) Evaluating effects of methyldopa. Pennsylvania Medicine, 69, 25.

JENKINSON, D.H. (1973) Classification and properties of peripheral adrenergic receptors. British Medical Bulletin, $29,142$.
LUNDBAEK, K. (1962) Intravenous glucose tolerance as a tool in definition and diagnosis of diabetes mellitus. British Medical Journal, 1, 1507.

McLellan, G.H. (1971) Automated colorimetric method for estimating serum triglycerides. Clinical Chemistry, 17, 535.

Noble, R.P. \& CAMPBelL, F.M. (1970) Improved accuracy in automated fluorometric determination of plasma triglycerides. Clinical Chemistry, 16, 166.

Stone, M.C. \& Thorpe, J.M. (1966) A new technique for the investigation of the low-density lipoproteins in health and disease. Clinica Chimica Acta, 14, 812.

Trout, D.L., Estes, E.H. JR \& FriedberG, S.J. (1960) Titration of free fatty acids of plasma: a study of current methods and a new modification. Journal of Lipid Research, 1, 199.

Woodman, D.D. \& Price, C.P. (1972) Estimation of serum total lipids. Clinica Chimica Acta, 38, 39. 